\title{
IGR J17254-3257, a new bursting neutron star
}

\author{
J. Chenevez ${ }^{1}$, M. Falanga ${ }^{2,3}$, E. Kuulkers ${ }^{4}$, R. Walter ${ }^{5,6}$, L. Bildsten ${ }^{7}$, S. Brandt ${ }^{1}$, N. Lund ${ }^{1}$, \\ T. Oosterbroek ${ }^{8}$, and J. Zurita Heras ${ }^{2}$
}

\author{
1 Danish National Space Center, Technical University of Denmark, Juliane Maries Vej 30, 2100 Copenhagen, Denmark \\ e-mail: jerome@spacecenter.dk \\ 2 CEA Saclay, DSM/DAPNIA/Service d'Astrophysique (CNRS FRE 2591), 91191, Gif-sur-Yvette, France \\ 3 Unité mixte de recherche Astroparticule et Cosmologie, 11 place Berthelot, 75005 Paris, France \\ 4 ISOC, ESA/ESAC, Urb. Villafranca del Castillo, PO Box 50727, 28080 Madrid, Spain \\ INTEGRAL Science Data Centre, Chemin d'Écogia 16, 1290 Versoix, Switzerland \\ 6 Observatoire de Genève, Université de Genève, 51 ch. des Maillettes, 1290 Sauverny, Switzerland \\ 7 Kavli Institute for Theoretical Physics, Kohn Hall, University of California, Santa Barbara, CA 93106, USA \\ 8 Science Payload and Advance Concepts Office, ESA-ESTEC, Postbus 299, 2200 AG, Noordwijk, The Netherlands \\ Received 16 March 2007 / Accepted 3 May 2007
}

\section{ABSTRACT}

\begin{abstract}
Aims. The study of the observational properties of uncommonly long bursts from low luminosity sources is important when investigating the transition from a hydrogen - rich bursting regime to a pure helium regime and from helium burning to carbon burning as predicted by current burst theories. On a few occasions X-ray bursts have been observed with extended decay times up to several tens of minutes, intermediate between usual type I X-ray bursts and so-called superbursts.

Methods. IGR J17254-3257 is a recently discovered X-ray burster of which only two bursts have been recorded: an ordinary short type I X-ray burst, and a 15 min long burst. The properties of the X-ray bursts observed from IGR J17254-3257 are investigated. The broad-band spectrum of the persistent emission in the $0.3-100 \mathrm{keV}$ energy band is studied using contemporaneous INTEGRAL and $X M M$-Newton data.

Results. A refined position of IGR J17254-3257 is given and an upper limit to its distance is estimated to about $14.5 \mathrm{kpc}$. The persistent bolometric flux of $1.1 \times 10^{-10} \mathrm{erg} \mathrm{cm}^{-2} \mathrm{~s}^{-1}$ corresponds, at the canonical distance of $8 \mathrm{kpc}$, to $L_{\mathrm{pers}} \approx 8.4 \times 10^{35} \mathrm{erg} \mathrm{s}^{-1}$ between 0.1-100 keV, which translates to a mean accretion rate of about $7 \times 10^{-11} M_{\odot} \mathrm{yr}^{-1}$.

Conclusions. The low X-ray persistent luminosity of IGR J17254-3257 seems to indicate the source may be in a state of low accretion rate usually associated with a hard spectrum in the X-ray range. The nuclear burning regime may be intermediate between pure $\mathrm{He}$ and mixed $\mathrm{H} / \mathrm{He}$ burning. The long burst is the result of the accumulation of a thick He layer, while the short one is a prematurate H-triggered He burning burst at a slightly lower accretion rate.
\end{abstract}

Key words. binaries: close - stars: individual: IGR J17254-3257 (1RXS J172525.5-325717) - stars: neutron - X-rays: bursts

\section{Introduction}

The X-ray source IGR J17254-3257 was discovered with the International Gamma-Ray Astrophysics Laboratory (INTEGRAL) in the Galactic Centre hard X-ray survey and was reported at $11 \sigma$ detection in a $20-60 \mathrm{keV}$ mosaic accumulated between February 27 and October 19, 2003 (Walter et al. 2004). From the derived source coordinates, IGR J17254-3257 was tentatively identified as the ROSAT source 1RXS J172525.5325717 (Stephen et al. 2005). However, little was known about the nature of the compact source and the system. An ordinary type I X-ray burst which occurred on February 17, 2004 with a peak flux of $0.8 \mathrm{Crab}$ at $3-30 \mathrm{keV}$ has been detected in INTEGRAL/JEM-X archival data (Brandt et al. 2006).

We found a second burst of IGR J17254-3257 lasting about fifteen minutes in INTEGRAL observations of the Galactic Centre performed on October 1st 2006. Only on a few occasions have type I X-ray bursts shown decay times ranging between ten and a few tens of minutes. Known examples of such observations are 4U 1724-307 (Swank et al. 1977), 4U 170823 (e.g., Hoffman et al. 1978), GX 17+2 (Kuulkers et al. 2002), SLX 1737-282 (in 't Zand et al. 2002), SLX 1735-269 (Molkov et al. 2005), 2S 0918-549 (in 't Zand et al. 2005), and GX 3+1 (Chenevez et al. 2006). These long bursts have durations and energy releases $\left(\sim 10^{41} \mathrm{erg}\right)$ intermediate between usual type I $\mathrm{X}$-ray bursts and so-called superbursts which last more than an hour (e.g., Kuulkers 2004). The mechanisms driving such long bursters at very low persistent luminosity have been the subject of recent investigations (e.g., Peng et al. 2007; Cooper \& Narayan 2007), suggesting that thermally unstable hydrogen ignition results in sporadic energetic helium bursts in a mixed hydrogen and helium environment. However, long helium bursts are also observed at low pure helium accretion rates (e.g., in 't Zand et al. 2005, 2007). IGR J17254-3257 thus provides a new example of these few faint persistent neutron stars (NS) that display type I X-ray bursts of such different durations.

In this letter we report the INTEGRAL/JEM-X results of the light curve and spectral analysis of the short and long type I bursts of IGR J17254-3257, hereafter referred to as burst 1 and burst 2, respectively. Using XMM-Newton $(0.3-10 \mathrm{keV})$ and INTEGRAL (5-100 keV) data we also study the broad-band spectrum of the persistent emission of this source.

\section{Data analysis and results}

Burst 1 and burst 2 have been discovered in INTEGRAL/JEM$\mathrm{X}$ (Winkler et al. 2003; Lund et al. 2003) 3-20 keV data, on 


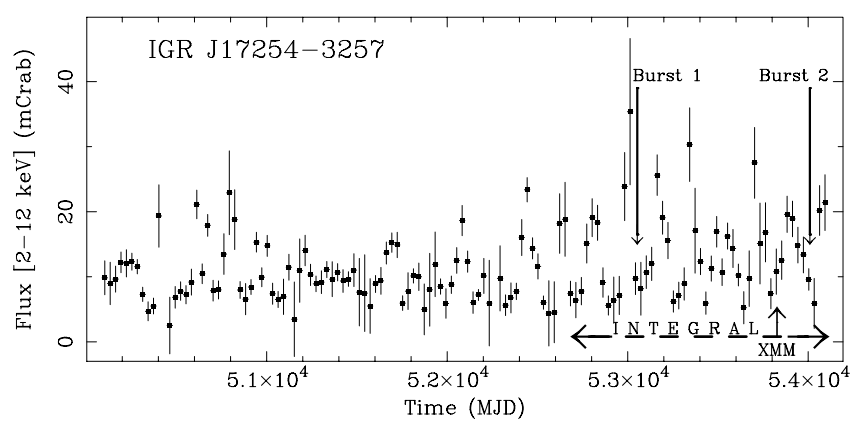

Fig. 1. RXTE/ASM light curve for IGR J17254-3257 averaged over 30-day intervals from January 6, 1996. The count rate has been converted into flux using $1 \mathrm{Crab}$ Unit for $75 \mathrm{cts} \mathrm{s}^{-1}$ (Levine et al. 1996). The times of burst 1 and burst 2, as well as the present data set coverage, are indicated by arrows.

February 17, 2004 and October 1st, 2006, respectively. In the IBIS/ISGRI (Ubertini et al. 2003; Lebrun et al. 2003) $18-40 \mathrm{keV}$ energy band neither of the two bursts were detected at a statistically significant level.

The persistent broad-band emission $(0.3-100 \mathrm{keV})$ of IGR J17254-3257 is obtained from INTEGRAL/JEM-X (3-20 keV) and IBIS/ISGRI (20-100 keV) data from March 2003 to October 2006, as well as XMM-Newton (Jansen et al. 2001) data acquired on April 2, 2006 (MJD 53827) for about $10 \mathrm{ks}$. The INTEGRAL data were extracted for all pointings within $3^{\circ}$ (JEM-X) and $4^{\circ}$ (ISGRI) of the source position for a total effective exposure (taking account of instrumental effects) of about $400 \mathrm{ks}$ and $830 \mathrm{ks}$, respectively. To study the (weak) persistent X-ray emission, the JEM-X and ISGRI spectra have been derived from mosaic images in six energy bands for JEM-X (3-20 keV) and four energy bands for ISGRI (20-100 keV). The data reduction was performed using the standard Offline Science Analysis (OSA) software version 6.0. A systematic error of $2 \%$ was applied to JEM-X and ISGRI spectra, which corresponds to the current uncertainties in the response matrices. All uncertainties in the spectral parameters are given at a $90 \%$ confidence level for single parameters.

We used 0.3-12 keV XMM-Newton data from the EPIC -pn (Strüder et al. 2001) and -MOS1 cameras (Turner et al. 2001) operating in full window mode. The data reduction and analysis were done with the XMM-Newton Science Analysis System (SAS version 7.0). These data allowed us to derive a refined source position at $\alpha_{\mathrm{J} 2000}=17^{\mathrm{h}} 25^{\mathrm{m}} 24^{\mathrm{s}} .8$ and $\delta_{\mathrm{J} 2000}=-32^{\circ} 57^{\prime} 15^{\prime \prime}$ with an uncertainty of $2^{\prime \prime}$. This is still inside the ROSAT error box of 1RXS J172525.5-325717, but it excludes the optical counterpart suggested by Stephen et al. (2005); no other known optical or infrared counterpart is found in the XMM-Newton error circle.

\subsection{Persistent emission}

In Fig. 1 we show the $2-12 \mathrm{keV}$ persistent emission for IGR J17254-3257 obtained with RXTE/ASM (Levine et al. 1996). The source is a weak persistent X-ray source. Since the persistent emission of IGR J17254-3257 is more or less stable over the whole available data set, its broad-band spectrum has been obtained by combining the XMM-Newton and INTEGRAL observations.

The joined pn/MOS1/JEM-X/ISGRI spectrum is best fit with a photoelectrically-absorbed blackbody (BB) and cutoff power-law (PL) model (Fig. 2). A multiplicative factor for each instrument was included in the fit to take account of the

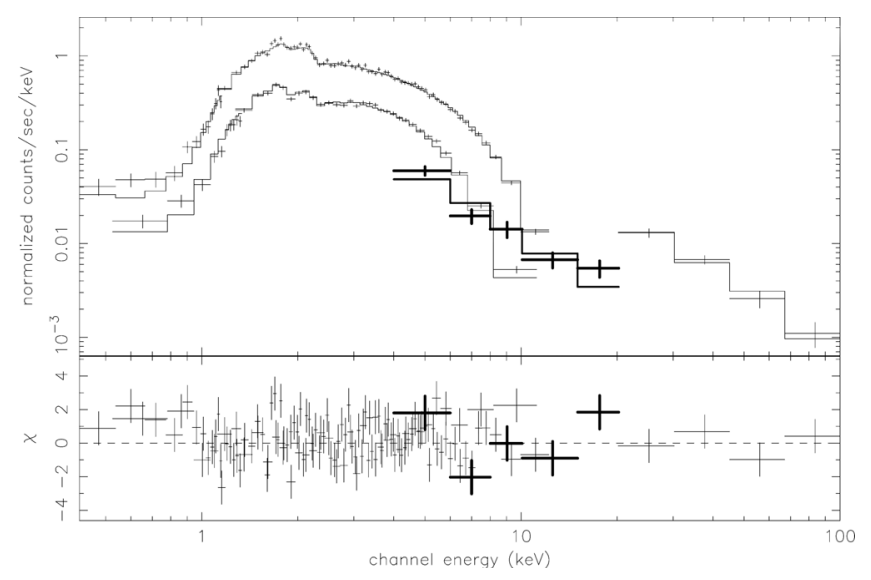

Fig. 2. The persistent spectrum of IGR J17254-3257 fit with a photoelectrically-absorbed blackbody (BB) plus a cutoff power-law (PL) model. The data points correspond to the EPIC- pn $(0.3-12 \mathrm{keV})$, MOS1 (0.5-10 keV), JEM-X (5-20 keV - bold), and ISGRI (20-100 keV) spectra, respectively. The lower panel shows the residuals between the data and the model.

uncertainty in the cross-calibration of the instruments. The factor was fixed at 1 for the XMM-Newton/EPIC-pn data and the normalizations of the MOS1, JEM-X and ISGRI data were found within $0.89 \pm 0.04$. The best fit parameters, with $\chi^{2} /$ d.o.f. $=$ $716 / 697$, are: absorption $N_{\mathrm{H}}=1.79_{-0.1}^{+0.08} \times 10^{22} \mathrm{~cm}^{-2}$, BB temperature $k T_{\mathrm{bb}}=1.06_{-0.1}^{+0.08} \mathrm{keV}$, a PL photon index $\Gamma=1.64_{-0.1}^{+0.07}$, and cutoff energy $E_{\mathrm{c}}=62_{-24}^{+24} \mathrm{keV}$. The $0.3-100 \mathrm{keV}$ unabsorbed flux is $10^{-10} \mathrm{erg} \mathrm{cm}^{-2} \mathrm{~s}^{-1}$. Note that, if we replace the soft thermal emission by a multi-temperature disc BB model (Mitsuda et al. 1984), we find the same $\chi^{2}$ value with a higher $k T_{\mathrm{bb}} \approx 1.6 \mathrm{keV}$, but the inner disk radius, $R_{\text {in }} \sqrt{\cos i}=0.4_{-0.1}^{+0.1} \mathrm{~km}$ (at $8 \mathrm{kpc}$ ) is smaller than the expected NS radius.

\subsection{Burst light curves}

In Figs. 3 and 4 we show the JEM-X 3-20 keV light curves for bursts 1 and 2, respectively. Burst 2 was observed during two consecutive stable pointings with a 2 minute satellite slew in between. Since the position of IGR J17254-3257 did not traverse the field of view, as well as the background due to other sources did not significantly evolve during the slew, it was possible to obtain the light curve during this interval by a simple linear interpolation of the detector event list. Taking account of the slight illumination increase due to the apparent source position change between the two pointings, we renormalized the slew burst count rate to the background-subtracted light curve during the stable pointings for the same time bin and energy band. The slew time interval is shown in Fig. 4 between the vertical dashed lines.

Both bursts were soft and no emission was detected above $20 \mathrm{keV}$. The start time for each burst was determined when the intensity rose to $10 \%$ of the peak above the persistent intensity level. The rise time is defined as the time between the start of the burst and the time at which the intensity reached $90 \%$ of the peak burst intensity. For burst 1 it was $2 \pm 1 \mathrm{~s}$; the e-folding decay time was $15.7 \pm 6.7 \mathrm{~s}$ and the total duration was about $29 \mathrm{~s}$. Burst 2 was about half as intense as burst 1 . However, its total duration was at least $900 \mathrm{~s}$ with a rise time of $20 \pm 5 \mathrm{~s}$ and an e-folding decay time of $219 \pm 32 \mathrm{~s}$. In both cases the persistent emission before and after the burst interval was not significantly detected in neither JEM-X nor ISGRI. 


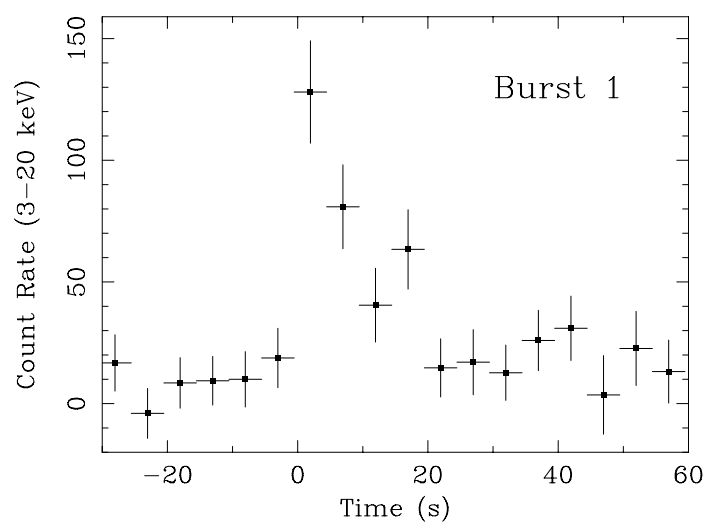

Fig. 3. The short type I X-ray burst detected from IGR J17254-3257 on February 17, 2004. Time 0 corresponds to 19:44:00 (UTC). The JEM-X (3-20 keV) net light curve is shown (background subtracted) with a time bin of $5 \mathrm{~s}$.

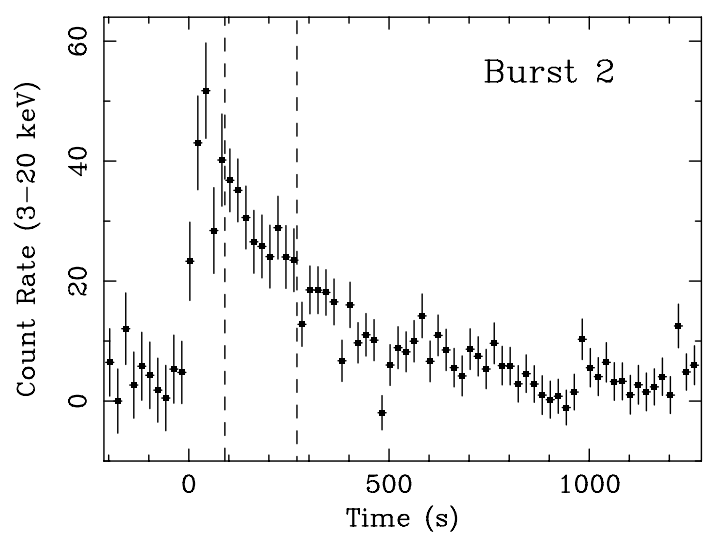

Fig. 4. Same as Fig. 3 for the long burst on October 1st, 2006 with a time bin of $20 \mathrm{~s}$. Time 0 corresponds to 7:13:37 (UTC). The vertical dashed lines indicate the time of the INTEGRAL slew interval.

\subsection{X-ray burst spectra}

For the spectral analysis of the bursts we used JEM-X data in the 3-20 keV band. For burst 1 we only performed a time-averaged (29 s) spectral analysis, due to its short duration. The net burst spectrum was well fit $\left(\chi_{\text {red }}^{2}=1.2\right)$ by a simple BB model.

For the long burst 2 we analyzed the spectrum during the $95 \mathrm{~s}$ interval ("peak") and the $645 \mathrm{~s}$ interval ("decay") before and after the slew, respectively. This allows to measure the thermal cooling during the decay, which is one of the charateristics of type I X-ray bursts. Indeed, though the uncertainties of the spectral fits do not indicate a significant softening during the decay, the corresponding 3-6 keV/6-20 keV hardness does decrease by a factor 2 . Finally, we determined the time-averaged (740 s) spectrum, excluding the slew interval. In each case, the same model as for burst 1 was used.

The inferred $\mathrm{BB}$ temperature, $k T_{\mathrm{bb}}$, and apparent $\mathrm{BB}$ radius at $8 \mathrm{kpc}, R_{\mathrm{bb}}$, for both bursts are reported in Table 1 . The burst fluences are obtained from the bolometric fluxes, $F_{\text {bol }}$, extrapolated in the $0.1-100 \mathrm{keV}$ energy range and integrated over the respective burst durations. The peak fluxes, $F_{\text {peak }}$, are derived from the peak count rates in fine time resolution and renormalized for the same energy range.
Table 1. Analysis results of the two bursts.

\begin{tabular}{lllll}
\hline \hline Dataset & \multirow{2}{*}{$\begin{array}{l}\text { Burst 1 } \\
\text { Parameters }\end{array}$} & \multicolumn{3}{c}{ Burst 2 } \\
\cline { 3 - 5 }$k T_{\mathrm{bb}}(\mathrm{keV})$ & $1.4_{-0.4}^{+0.5}$ & $1.6_{-0.2}^{+0.3}$ & $1.2_{-0.2}^{+0.3}$ & $1.3_{-0.2}^{+0.2}$ \\
$R_{\mathrm{bb}, \mathrm{d}_{\text {gkpc }}}(\mathrm{km})$ & $12_{-6}^{+13}$ & $6.4_{-4}^{+3}$ & $5.6_{-2}^{+4}$ & $5.1_{-2}^{+2}$ \\
$\chi^{2} /$ d.o.f. & $12 / 10$ & $48 / 49$ & $48 / 42$ & $59 / 47$ \\
$F_{\text {bol }}{ }^{+2}$ & 8.9 & 4.9 & 1.0 & 1.1 \\
\hline Burst parameters & & & \\
$F_{\text {peak }}{ }^{a}$ & $\simeq 20$ & & $\simeq 12$ & \\
$f_{\mathrm{b} b}{ }^{c}$ & $2.6 \times 10^{-7}$ & & $2.6 \times 10^{-6}$ \\
$\tau^{c}$ & 13 & & 216 \\
$\gamma^{d}$ & 0.006 & & 0.009 \\
\hline
\end{tabular}

a Unabsorbed flux (0.1-100 keV) in units of $10^{-9} \mathrm{erg} \mathrm{cm}^{-2} \mathrm{~s}^{-1}$. ${ }^{b}$ Fluence $\left(\mathrm{erg} \mathrm{cm}^{-2}\right){ }^{c} \tau(\mathrm{s}) \equiv f_{\mathrm{b}} / F_{\text {peak }} \cdot{ }^{d} \gamma \equiv F_{\text {pers }} / F_{\text {peak }} ; F_{\text {pers }}=$ $1.1 \times 10^{-10} \mathrm{erg} \mathrm{cm}^{-2} \mathrm{~s}^{-1}(0.1-100 \mathrm{keV})$.

\section{Discussion and conclusions}

We have found two X-ray bursts of very different durations from IGR J17254-3257. The first one is an ordinary short type I X-ray burst while the second one is similar to the other few intermediately long bursts reported to date. Assuming that the bolometric peak luminosity of burst 1 was at most at the Eddington limit for a helium burst, $L_{\mathrm{Edd}} \approx 3.8 \times 10^{38} \mathrm{erg} \mathrm{s}^{-1}$, as empirically derived by Kuulkers et al. (2003), we can derive an upper limit to the distance of the source of about $14.5 \mathrm{kpc}$. This is also consistent with the high measured column density, which is a factor 2 higher than the total galactic column density as estimated from HI maps in that direction (Dickey \& Lockman 1990).

The best fit to the broad-band $0.3-100 \mathrm{keV}$ persistent emission spectrum of IGR J17254-3257 required a two-component model: a cutoff PL together with a BB soft component; the hard spectral component contributes most to the observed flux (95\%). These spectral characteristics are similar to those observed in the low/hard state of low-mass X-ray binaries (LMXB) weakly magnetized NS (see, e.g., Barret et al. 2000; Falanga et al. 2006). The soft thermal emission, $k T_{\mathrm{bb}}$, could be associated with the radiation from the hot-spot on the NS surface around an accretion shock. Assuming a canonical distance of $8 \mathrm{kpc}$ for a LMXB in the direction of the Galactic Centre, the estimated persistent flux between $0.1-100 \mathrm{keV}, F_{\text {pers }}=1.1 \times 10^{-10} \mathrm{erg} \mathrm{cm}^{-2} \mathrm{~s}^{-1}$, translates to a bolometric luminosity $L_{\text {pers }} \approx 8.4 \times 10^{35} \mathrm{erg} \mathrm{s}^{-1}$. This makes IGR J17254-3257 another member of the class of bursters with low persistent emission (see, e.g., Cocchi et al. 2001; Cornelisse et al. 2004, and references therein). The mass accretion rate per unit area of the persistent emission, given by $L_{\text {pers }} \eta^{-1} \mathrm{c}^{-2} / 4 \pi R_{\mathrm{NS}}^{2}$ (where $\eta \simeq 0.2$ is the accretion efficiency for a $1.4 M_{\odot}$ and $10 \mathrm{~km}$ radius NS), is $\dot{m} \approx 370 \mathrm{~g} \mathrm{~cm}^{-2} \mathrm{~s}^{-1}$. Since the ASM light curve does not indicate strong differences in the persistent flux of the source at the time of the two bursts, we are not able to comment on the exact accretion state at any time. We assume therefore that both bursts occurred at about the same accretion rate. From the detection of only two bursts during the total observation time of about $860 \mathrm{ks}$ elapsed on the source by JEM-X and $X M M-N e w t o n$, we can estimate a recurrence time of five days.

Both bursts are well described by a simple BB model representing the thermal emission from the neutron star surface, which is consistent with the observed properties of type I X-ray bursts (see, e.g., Galloway et al. 2006). With a fast rise and exponential decay light curve burst 1 is indeed similar to commonly observed normal type I bursts. Its total energy release was 
$E_{b, 1} \simeq 2 \times 10^{39} \mathrm{erg}$ (assuming $8 \mathrm{kpc}$ distance), not that large considering the inferred long accumulation time. The 15 minute duration of burst 2 is more unusual. The total energy release $E_{b, 2} \simeq 2 \times 10^{40} \mathrm{erg}$ (at $8 \mathrm{kpc}$ distance) corresponds to an ignition column $y=E_{b, 2}(1+z) / 4 \pi R_{\mathrm{NS}}^{2} Q_{\text {nuc }}$ ranging between $y \approx 5 \times 10^{8} \mathrm{~g} \mathrm{~cm}^{-2}$ for burning hydrogen with abundance $X=0.7$, and $y \approx 13 \times 10^{8} \mathrm{~g} \mathrm{~cm}^{-2}$ for $X=0$ (pure helium); here $Q_{\text {nuc }}=1.6+4 X \mathrm{Mev}$ nucleon ${ }^{-1}$ is the nuclear energy release for a given average hydrogen fraction at ignition $X$, and $z=0.31$ is the appropriate gravitational redshift at the surface of a $1.4 M_{\odot}$ NS.

Interestingly, in 't Zand et al. (2007) have recently classified IGR J17254-3257 as a new candidate ultracompact X-ray binary, explaining its low luminosity by a small accretion disk. In the same category we find the burster 2S 0918-549 from which the observation of both short bursts and a 40 min long burst (in 't Zand et al. 2005) indeed makes this source quite similar to IGR J17254-3257. Suggesting the companion of 2S 0918-549 is probably a helium white dwarf, in 't Zand et al. (2005) showed that its long burst is consistent with pure helium ignition and explained the different burst durations as related to the ignition thickness and to changes in the composition of the layer between the bursts. However, the composition of the accreted material in IGR J17254-3257 may not necessarily be pure helium. The presence of some hydrogen may indeed explain what distinguishes burst 2 from the long burst of 2S 0918-549: its relative weakness with respect to the Eddington limit, while the long burst from 2S 0918-549 did apparently reach this limit, and its relatively long rise time.

As a matter of fact, the inferred mass accretion rate of IGR J17254-3257 is near the value where the accumulating hydrogen transitions from unstable burning at low accretion rates, to stable burning (via the Hot CNO cycle) at higher accretion rates (e.g. Strohmayer \& Bildsten 2004). When stable, the hydrogen burning steadily accumulates a thick helium shell that eventually ignites. Indeed, the lower limit to the burst 2 recurrence time of a few tens of days is in the range of those predicted by helium ignition models (see Table 2 of Cumming $\&$ Bildsten 2000). Pure helium bursts of such thick columns also lead to long burst durations (e.g., Cumming et al. 2006; Peng et al. 2007). Moreover, Cumming \& Bildsten (2000) show that for pure helium ignition, the ignition column is very sensitive to the accretion rate. In particular, the transition to unstable hydrogen burning can be quite sudden, leading to short mixed $\mathrm{H} / \mathrm{He}$ bursts. As shown by Cooper \& Narayan (2007) both energetic pure helium flashes and weak hydrogen flashes may occur near the transition. These weak hydrogen bursts (undetectable because their peak luminosity is lower than the persistent luminosity) contribute to the building of the deep layer of nearly pure helium and of which they may trigger the ignition if the mass of helium is sufficiently large. Our burst 1 is indeed similar to a $\sim 10 \mathrm{~s}$ decay helium burst triggered by hydrogen ignition as simulated by Peng et al. (2007) sedimentation model at low accretion rates (see Fig. 6 of Peng et al. 2007).

We conclude that the long burst of IGR J17254-3257 results from the ignition of a large helium pile beneath a steady hydrogen burning shell, while the short event occured at a slightly lower accretion rate where a weak hydrogen flash prematurely triggered a mixed $\mathrm{H} / \mathrm{He}$ burning burst.

Acknowledgements. J.C. acknowledges financial support from ESA-PRODEX. M.F. acknowledges the French Space Agency (CNES) for financial support.

\section{References}

Barret, D., Olive, J. F., Boirin, L., et al. 2000, ApJ, 533, 329

Brandt, S., Budtz-Joergensen, C., \& Chenevez, J. 2006, Astr. Tel., 778

Chenevez, J., Falanga, M., Brandt, S., et al. 2006, A\&A, 449, L5

Cocchi, M., Bazzano, A., NataLucci, L., et al. 2001, A\&A, 378, L71

Cooper, R., \& Narayan, R. 2007, ApJ, 661, 468

Cornelisse, R., in 't Zand, J. J. M., Kuulkers, E., et al. 2004, Nucl. Phys. B (Proc. Suppl.), 132, 518

Cumming, A., \& Bildsten, L. 2000, ApJ, 544, 453

Cumming, A., Macbeth, J., \& in 't Zand, J. J. M., \& Page, D. 2006, ApJ, 646, 429

Dickey, J. M., \& Lockman, F. J. 1990, ARA\&A, 28, 215

Falanga, M., Götz, D., Goldoni, P., et al. 2006, A\&A, 458, 21

Galloway, D. K., Muno, M. P., Hartman, J. M., et al. 2006, ApJS, submitted [arXiv:astro-ph/0608259]

Hoffman, J. A., Lewin, W. H. G., Doty, J., et al. 1978, ApJ, 221, L57

in 't Zand, J. J. M., Verbunt, F., Kuulkers, E., et al. 2002, A\&A, 389, L43

in 't Zand, J. J. M., et al. 2005, A\&A, 441, 675

in 't Zand, J. J. M., Jonker, P. G., \& Markwardt, C. B. 2007, A\&A, 465, 953

Jansen, A., Lumb, D., Altieri, B., et al. 2001, A\&A, 365, L1

Kuulkers, E. 2004, Nucl. Phys. B, 132, 466

Kuulkers, E., Homan, J., van der Klis, M., et al. 2002, A\&A, 382, 947

Kuulkers, E., den Hartog, P. R., in't Zand, J. J. M., et al. 2003, A\&A, 399, 663

Lebrun, F., Leray, J.-P., Lavocate, Ph., et al. 2003, A\&A, 411, L141

Levine, A. M., Bradt, H., \& Cui, W. 1996, ApJ, 469, L33

Lund, N., et al. 2003, A\&A, 411, L231

Mitsuda, K., Inoue, H., Koyama, K., et al. 1984, PASJ, 36, 741

Molkov, S., Revnivtsev, M., Lutovinov, A., \& Sunyaev, R. A. 2005, A\&A, 434, 1069

Peng, F., Brown, E. F., \& truran, J. W. 2007, ApJ, 654, 1022

Stephen, J. B., Bassani, L., Molina, M., et al. 2005, A\&A, 432, L52

Strohmayer, T. E., \& Bildsten, L. 2004, in Compact stellar X-ray sources, ed. W. H. G. Lewin, \& M. van der Klis (Cambridge: Cambridge University Press) [arXiv: astro-ph/0301544]

Strüder, L., Briel, U., Dennerl, K., et al. 2001, A\&A, 365, L18

Swank, J. H., Becker, R. H., Boldt, E. A., et al. 1977, ApJ, 212, L73

Turner, M. J. L., Abbey, A., Arnaud, M., et al. 2001, A\&A, 365, L27

Ubertini, P., Lebrun, F., Di Cocco, G., et al. 2003, A\&A, 411, L131

Walter, R., Bodaghee, A., \& Barlow, E. J. 2004, Astr. Tel., 229

Winkler, C., Courvoisier, T. J.-L., et al. 2003, A\&A, 411, L1 